\title{
Os Problemas Mais Graves e Mais Freqüentes no Uso dos Testes Psicológicos
}

\author{
Ana Paula Porto Noronha
}

Universidade São Francisco

\begin{abstract}
Resumo
Considerando a importância da Avaliação Psicológica na atuação profissional do psicólogo, este estudo objetivou identificar os problemas mais graves e mais freqüentes no uso dos testes psicológicos, segundo a concepção de psicólogos e listar os instrumentos mais utilizados pelos psicólogos. Participaram como sujeitos do estudo 214 psicólogos inscritos no CRP / $6^{a}$ Região e o material utilizado foi um questionário enviado pelo correio. Os resultados demostraram que: 1) segundo os psicólogos, os problemas mais graves no uso dos testes psicológicos são os relativos aos próprios instrumentos e ao seu uso; 2) os problemas mais freqüentes no uso dos testes psicológicos, segundo os sujeitos, são os relativos ao instrumento e à formação dos psicólogos. Concluiu-se que é necessário o estabelecimento de parâmetros específicos para a formação na área.

Palavras-chaves: Avaliação psicológica; formação profissional; testes psicológicos.
\end{abstract}

The Worst and the Most Common Problems in the Use of the Psychological Tests

Abstract

Considering the importance of the Psychological Assessment in the psychologist's professional field, this study had the following goals: to identify the worst and the most commom problems in the use of the psychological tests and to list the most tests used by the subjects sample. The subjects of this study were 214 licensed psychologists from the Regional Council Section $6^{a}$ and the instrument used was a questionnaire sent to them by mail. Results showed that: 1) according to the psychologists, the worst problems in the use of the tests are related to the instruments themselves and its use; 2) the most frequent problems in the use of the psychological tests, according to the subjects, are related to the instrument and to the academic preparation of psychologists. Conclusion indicates that there is a crucial need to establish parameters to improve the academic preparation in this area.

Keywords: Psychological assessment; academic preparation; psychological tests.

\section{A Avaliação Psicológica e os Testes Psicológicos}

A avaliação psicológica é uma atividade profissiona bastante questionada e controvertida na Psicologia. A avaliação não teve um início que lhe conferisse simpatia: começou com a rotulação dos doentes e débeis mentais e foi muito criticada por seus métodos psicofísicos e pela pouca precisão científica de seus resultados (Anastasi \& Urbina, 2000; Ancona-Lopez, 1987).

Durante muitos anos esta prática sofreu as conseqüências de seu desenvolvimento conturbado; mas, encontra-se hoje em um momento de modificação. A avaliação continua a ser questionada e é natural que o seja; tal questionamento verifica-se em todas as áreas do conhecimento, sendo útil e necessário, pois gera estudos

Endereço para correspondência: Rua Alexandre Rodrigues Barbosa, 45, Centro, Itatiba, SP, 13.250-000. E-mail: ananoronha@saofrancisco.edu.br e pesquisas. No que tange à avaliação, seu estudo é válido para reconhecer-lhe a importância e valorizá-la.

Para Pasquali (1992), as controvérsias e os questionamentos da avaliação psicológica continuam e continuarão Mas é necessário, apoiando umas, superar outras que são devidas a confusões e incompreensões de várias naturezas.

Ainda são presentes os questionamentos acerca da necessidade, ou não, da realização da avaliação psicológica em determinadas áreas de atuação profissional. De qualquer forma, parece inconcebível o fato de que as intervenções ocorram sem que as tenham precedido as avaliações, já que ela é fundamental para uma atuação adequada, como discutem muitos autores, dentre eles, Aftanas (1994), que em seu estudo conclui que a avaliação psicológica deve ser considerada como uma necessidade primária. Para Witter e David (1996), a avaliação de uma maneira geral, seja ela psicológica, ou não, é de extrema relevância para a tomada de decisões, ou seja, a avaliação serve para orientar uma ação mais segura e adequada do psicólogo no seu trabalho. 
No entanto, não parece ser possível estabelecer uma concordância entre a comunidade psicológica, no que diz respeito aos métodos e às técnicas utilizadas, assim como ao tempo previsto para a realização da avaliação, e aos procedimentos, pois considerando que a Psicologia é uma ciência em que muitas orientações teóricas e leituras de homem são possíveis, e considerando ainda, que há dentro da psicologia uma grande variedade de contextos de atuação do psicólogo, o que exige dele diferentes posturas de acordo com as necessidades específicas, certamente diferentes processos avaliativos são necessários. É compreensível que de tal subdivisão, decorram diferentes estilos e posturas profissionais e essas diferenças podem trazer contribuições para a área, à medida que estimulam reflexões, discussões e críticas; acredita-se, desta forma, que o resultado disto seja positivo e gere o desenvolvimento.

Muitos são os estudos e as pesquisas que geram discussões a respeito da Avaliação Psicológica. Azevedo, Almeida, Pasquali e Veiga (1996) apontam que o baixo teor científico dos instrumentos padronizados vem sendo veementemente denunciado. Esses autores discutem que o fato de estudos na área estarem sendo realizados revela uma melhor reputação da investigação psicológica e do uso de instrumentos padronizados. Apontam também para os últimos estudos que partem do princípio de que a avaliação psicológica é indispensável, e procuram destacar a melhora da qualidade dos instrumentos padronizados.

Problemas Relativos à Avaliação Psicológica e aos Testes Psicológicos

Segundo Wechsler (1999), o Brasil encontra-se na retomada dos estudos sobre Avaliação Psicológica. Para ela, nos últimos quinze anos, a avaliação psicológica sofreu um descrédito, em que os testes passaram a ser criticados por não serem adequados à realidade brasileira. Dentre os principais problemas apresentados pelos testes psicológicos, os autores destacam: definição pouco simples do que o instrumento mede, ou seja, a complexidade do pressuposto teórico que subsidia a construção do instrumento (Sacuzzo \& Johnson, 1995); dificuldade encontrada na habilidade do psicólogo para compreender os dados e para fazer relações entre os diversos resultados encontrados, através dos próprios testes ou de qualquer outra técnica utilizada (Bruno, 1995).

Para Pasquali (1991) parte da dificuldade pode ser atribuída ao descaso de nossos pesquisadores, que pouco se preocupam com as qualidades psicométricas dos instrumentos psicológicos Embora tal linha de pesquisa já venha fazendo parte dos laboratórios de avaliação psicológica de algumas universidades brasileiras, os avanços necessários ainda não foram conseguidos. Por outro lado, em outras realidades, este tipo de trabalho já vem obtendo os resultados esperados, no sentido de pesquisar os parâmetros psicométricos dos testes psicológicos, a fim de se obter instrumentos com melhor qualidade e mais confiáveis (Groth-Marnat, 1997; Messick, 1995; Reynolds, 1998; Zimiles, 1996).

Um destaque deve ser dado para o trabalho de Almeida, Prieto, Muñiz e Bartram (1998), que fizeram um estudo interessante a respeito do uso dos testes em alguns países - Portugal, Espanha e Ibero-Americanos (dentre eles o Brasil) - e chegaram à conclusão de que os problemas mais freqüentes na prática dos testes são: xerocar material de testes; usar testes inadequados para algumas situações; não estar em sintonia com as modificações da área; avaliações incorretas; não usar folhas de respostas padronizadas; não ter clareza das limitações dos instrumentos, quanto às normas; aplicação de testes por leigos; não adaptar os instrumentos para os determinados países ou regiões; não arquivar os instrumentos e não dar o devido seguimento aos estudos dos testes e fazer interpretações que extrapolam o instrumento.

Os autores salientam que não existem padrões de atuação na área, não se avalia competência profissional dos usuários de instrumento psicológico, não se determina uma conduta comum de formação específica, assim como não se estabelecem cursos de formação. Eles relacionam os problemas encontrados na formação profissional, com os problemas encontrados na utilização dos testes psicológicos. Em relação ao aspecto da xerocópia de material de testes citado no estudo anterior, o trabalho desenvolvido por Oakland (1999) também faz referência ao tema, atribuindo a ele o caráter de um dos problemas que impedem o desenvolvimento dos testes.

Outros estudos desta natureza têm sido realizados, como o de Halperin e McKay (1998), que procuraram revisar os testes psicológicos usados com crianças e adolescentes, na tentativa de responder como os testes vêm sendo usados e como seus escores estão sendo interpretados. Embora tenha sido reafirmado que os testes psicológicos são importantes instrumentos que podem contribuir com o plano de trabalho do profissional, o problema detectado girou em torno dos poucos dados estatísticos que o instrumento apresenta, sobretudo os testes projetivos. Isto é também enfatizado por Adánez (1999), uma vez que ele denuncia que os manuais mais clássicos da psicologia não tratam o assunto de maneira equilibrada, ou seja, priorizam a discussão de alguns elementos em detrimento de outros. Tal observação pode parecer descontextualizada, tendo em vista que fala dos manuais de psicologia, e não de 
manuais de testes, discussão que vinha sendo privilegiada até então. Por outro lado, já se ressaltou no estudo de Almeida e colaboradores (1998) que existe uma clara relação entre problemas nos instrumentos psicológicos e problemas na formação profissional do psicólogo que constrói e usa os referidos instrumentos.

Ainda dando margem a esta questão, da relação entre formação profissional e uso de instrumentos, parece claro que a formação de um psicólogo em cinco anos de universidade, não é suficiente para aprimorá-lo em todas as áreas de conhecimento, embora devesse sê-lo. No estudo realizado por Hays e Wellard (1998) a respeito da formação em avaliação psicológica, ficou evidente a necessidade de que o recém-formado continue os estudos na área após a graduação.

Pode-se entender que para os autores citados, os problemas encontrados na avaliação psicológica, e em especial nos testes psicológicos, referem-se prioritariamente à formação do profissional que utiliza os instrumentos, às deficiências nos próprios instrumentos, assim como à falta de pesquisas que promovam satisfatoriamente $\mathrm{O}$ desenvolvimento da área. Por trás deste cenário em que predominam controvérsias e polêmicas, existem duas preocupações básicas: como a avaliação tem sido entendida e utilizada pela comunidade profissional e como ela tem sido proposta e ensinada nos cursos preparatórios.

É importante que as pesquisas na área estejam preocupadas com a criação de instrumentos novos, com a atualização de instrumentos existentes, com a verificação dos parâmetros psicométricos dos instrumentos, assim como com a solução de problemas presentes na avaliação psicológica como um todo. Outros estudos desenvolvidos versam sobre quais são os instrumentos mais usados pelos psicólogos e os problemas mais presentes na avaliação, segundo esses profissionais (Almeida e colaboradores, 1998; Noronha, 1999).

Embora os avanços da avaliação sejam claros, sobretudo quando se relaciona a situação atual da área com os primeiros instrumentos psicofísicos, rudimentares e com falhas sérias, está clara a importância da continuidade de estudos e de pesquisas, a fim de que pesquisadores e psicólogos da avaliação psicológica encontrem o devido estado de excelência.

Face à importância da avaliação na prática profissional do psicólogo em qualquer contexto de trabalho e à necessidade de estudos atuais sobre esta área de conhecimento, o presente estudo teve como objetivos: identificar os problemas mais graves e mais freqüentes no uso dos testes psicológicos, segundo psicólogos; e listar os testes psicológicos mais utilizados.

\section{Método}

\section{Participantes}

Participaram como sujeitos desta pesquisa, 214 psicólogos sendo que $86,0 \%(n=184)$ eram do sexo feminino e $14 \%(n=30)$ do sexo masculino. A idade dos sujeitos variou de 39 a 76 anos, tendo sido a média de 48,2 anos e o desvio padrão 6,44. A maioria possui de 41 a 50 anos $(69,2 \%, n=148) ; 20,6 \%(n=44)$ possui de 51 a 60 anos; $5,1 \%(n=11)$ de 61 a 70 anos; $4,2 \%(n=9)$ de 39 a 40 anos e $0,9 \%(n=2)$ acima de 70 anos.

Todos os psicólogos estavam inscritos no $\mathrm{CRP} / \mathrm{\sigma}^{\mathrm{a}}$ Região e foram identificados por este órgão (sub-sede Campinas) de acordo com procedimentos próprios.

\section{Material}

O material utilizado no presente estudo constituiu-se de um questionário dividido em quatro partes, a saber: I. dados de identificação (informações relativas à caracterização do sujeito, no que diz respeito à idade, ao sexo, ao ano de formação e à pós-graduação); II. atuação profissional (informações relacionadas às atividades desenvolvidas pelos psicólogos, tais como áreas de atuação profissional e horas de trabalho dedicadas); III. testes psicológicos (informações dobre os nomes dos instrumentos padronizados mais utilizados na sua prática profissional, ano de publicação, autor); IV. Problemas relacionados à avaliação psicológica (informações relativas aos problemas mais graves e mais freqüentes na avaliação psicológica, segundo os psicólogos).

O material foi enviado pelo correio, juntamente com um envelope etiquetado para facilitar a devolução; foram enviados 3000 questionários e, dentro do período aprazado, foram recebidos 223. Destes, foram considerados $214(7,14 \%)$ para composição da amostra, tendo em vista que nove deles, apenas enviaram para justificar a não participação.

\section{Resultados}

De acordo com o primeiro objetivo deste trabalho, procurou-se investigar quais problemas os psicólogos apontam como graves e freqüentes em relação à área de Avaliação Psicológica. As respostas obtidas foram agrupadas em seis categorias, assim como as respostas em branco também foram computadas; as respostas foram submetidas à apreciação de dois especialistas na área (dois professores de disciplinas de Avaliação Psicológica: um doutor e um mestre). 
As categorias utilizadas nesta análise serão descritas a seguir:

Formação: nesta categoria foram incluídas todas as respostas relativas à formação profissional, tanto a básica (graduação), quanto à pós-graduação, tais como os relacionados às Instituições de Formação, aos órgãos de classe, aos docentes e aos próprios psicólogos. A seguir estão alguns exemplos de respostas classificadas nesta categoria "Falta de reciclagem profissional", "Psicólogo escondido atrás dos resultados dos testes", "Desconhecimento da base teórica".

Uso: foram incluídas as respostas que expressavam comentários à adequação ou inadequação de instrumentos de avaliação, tanto no que se refere à aplicação, avaliação ou interpretação dos resultados. Alguns exemplos de respostas classificadas nesta categoria: "Falta de clareza do que se está medindo", "Mau uso do material", "Uso limitado do instrumento e de suas possibilidades" e "Uso mecânico dos testes".

Instrumento: foram incluídas nesta categoria, respostas relativas aos problemas específicos dos instrumentos de avaliação mencionados, quer fossem relacionados construção deles, às suas características psicométricas, revisões, normas ou qualquer outro problema desta natureza. Encontram-se abaixo relacionados alguns exemplos de respostas: "Material antiquado", "Padronização estrangeira", "Crivos deixam a desejar" e "Faltam atualizações das normas".

Ético: nesta categoria foram relacionadas as questões éticas advindas de quaisquer dos princípios apontados no Código de Ética profissional do Psicólogo ou outros aspectos identificados pela prática dos sujeitos. Como exemplos: "Falta de ética na entrega dos resultados", "Testes conhecidos pelos candidatos", "Leigos têm acesso" e "Cópia do instrumento para diminuir custos".

Epistemológicos: foram incluídas nesta categoria respostas que tratavam de análises críticas dos princípios, hipóteses resultados da Ciência Psicológica, e em especial, da Avaliação
Psicológica e de seus instrumentos. Alguns exemplos de respostas: "Entendidos como verdades absolutas", "Pouca utilidade para prevenção", "Não medem a realidade" e "Fazem inferências".

Outras respostas: foram incluídas as respostas sem sentido ou com sentido dúbio e as respostas que não se enquadravam em nenhuma outra categoria. Como por exemplo, "Retirada do currículo obrigatório", "Não há material para avaliar crianças menores de cinco anos e adolescentes", "Autoritarismo técnico" e "Falta de coesão na nomenclatura".

Respostas em branco: pertencem a esta categoria os protocolos cujas perguntas não foram respondidas.

Observou-se que mais da metade de sujeitos não respondeu à questão - enumere os problemas mais graves no uso dos testes psicológicos - $(55,1 \%, n=118)$, o que pode indicar uma ausência de reflexões sobre este assunto. Deste total, $29,9 \%(n=64)$ justificaram que não responderiam porque não usavam testes psicológicos, e $25,2 \% \quad(n=54)$ deixaram a questão em branco (Tabela I).

Dos protocolos respondidos $(n=96)$, constatou-se que a categoria de maior freqüência de argumentos analisados foi instrumento $(32,9 \%, n=99)$, vindo em seguida, uso $(25,9 \%, n=78)$, formação $(19,3 \%, n=58)$, epistemológico $(10 \%, n=30)$, ético $(9,6 \%, n=29)$ e outras $(2,7 \%, n=7)$. Foi a categoria instrumento que recebeu mais crítica, representando o mais grave problema para a atuação em Avaliação Psicológica.

Foi realizado o teste de qui-quadrado para verificar a homogeneidade da distribuição; observou-se que houve diferença significativa entre as categorias $\left(X^{2} o=29,67\right.$; $X^{2} c=20,515$; n.g.l. 5 ; $\left.p<0,001\right)$, o que significa dizer que a hipótese de distribuição uniforme das respostas dos sujeitos foi rejeitada.

No que se refere aos problemas mais freqüentes dos instrumentos de avaliação psicológica, as categorias de respostas utilizadas para a análise foram as mesmas citadas na questão anterior, ou seja, formação, uso, instrumento, ético,

Tabela 1. Problemas Mais Graves e Mais Freqüentes no Uso dos Testes Psicológicos

\begin{tabular}{lcccccr}
\hline & Problemas Graves & \multicolumn{2}{c}{ Prob. Freqüentes } & \multicolumn{2}{c}{ Total } \\
\hline \multirow{2}{*}{ Formação } & $n$ & $\%$ & $n$ & $\%$ & $n$ & $\%$ \\
Uso & 58 & 19,3 & 90 & 27,5 & 148 & 23,5 \\
Instrumento & 78 & 25,9 & 64 & 19,5 & 142 & 22,6 \\
Ético & 99 & 32,9 & 124 & 37,8 & 223 & 35,5 \\
Epistemológico & 29 & 9,6 & 17 & 5,2 & 46 & 7,3 \\
Outros & 30 & 10 & 28 & 8,5 & 58 & 9,2 \\
& 7 & 23 & 5 & 1,5 & 12 & 1,9 \\
\hline
\end{tabular}


epistemológico, outras respostas, respostas em branco. A seguir, são apresentados alguns exemplos pertinentes às categorias: Formação: "Nem sempre é feita por profissionais capacitados", "Necessidade de treino" e "Conhecimento de poucos testes".

Uso: "Avaliação incorreta", "Falta de clareza da aplicação", "Erro de aplicação", "Erro de avaliação".

Instrumento: "Padrão deveria ser corrigido a cada 10/5 anos", Alto custo do material", "Faltam instrumentos para diferentes realidades", "Faltam amostras brasileiras".

Ético: "Muito divulgado na mídia", "Divulgação dos resultados a terceiros", "Popularização dos instrumentos", "Divulgação dos testes a leigos".

Epistemológico: "Pouca margem de flexibilidade às idiossincrasias", "Visão parcial do indivíduo", "Estigmatizam", "Catalogam".

Outras respostas: "Não tenho encontrado dificuldade", "O método é pouco difundido, facilitando panelinhas profissionais".

Em relação à discussão dos problemas mais freqüentes apontados pelos psicólogos, novamente 51,4\% dos sujeitos $(n=110)$ não responderam, sendo que $33,2 \%$ $(n=71)$ justificaram que não responderiam porque não utilizam testes psicológicos e 18,2\% $(n=39)$ deixaram a questão em branco. Dos protocolos respondidos, 37,8\% das respostas $(n=124)$ associaram os problemas mais freqüentes ao instrumento; $27,5 \%(n=90)$ relacionaram formação; $19,5 \%(n=64)$ ao uso; $8,5 \%(n=28)$ ao epistemológico; $5,2 \%(n=17)$ ao ético e $1,5 \%(n=5)$ a outras respostas.

Os sujeitos apontaram aspectos do instrumento como o problema mais freqüente em relação às categorias de respostas. Notou-se que houve diferença significativa entre as categorias $\left(X^{2} o=50,42 ; X^{2} c=20,515 ;\right.$ n.g.l. $5 ; p<0,001=$, o que significa dizer que a hipótese de distribuição igual entre as categorias de resposta foi rejeitada.

Em relação ao segundo objetivo do estudo, ou seja, verificar os instrumentos mais usados pelos psicólogos, observou-se que quando os sujeitos foram solicitados a se manifestar quanto aos dez instrumentos mais utilizados na prática profissional, apenas $43 \%(n=92)$ atenderam ao objetivo da questão, enquanto $39,3 \%$ dos sujeitos $(n=$ 84) disseram que não usavam testes na sua atuação e $17,7 \%(n=38)$ não responderam à pergunta e não fizeram comentário algum a respeito, deixando a questão em branco. Ao comparar os sujeitos quanto à utilização, ou não, dos testes psicológicos, observou-se que não houve diferença significante entre eles $\left(X^{2} o=11,2 ;\right.$ n.g.l. $\left.2 ; p>0,05\right)$.

Alguns dos argumentos explicativos dos sujeitos para o não uso dos testes, foram: "não uso testes, sou psicanalista"; "não tenho condições de responder, pois trabalho em treinamento"; "sou psicoterapeuta".

Quando se solicitou aos sujeitos que indicassem os instrumentos mais usados em sua prática, foram feitas 505 citações, sendo que em 83,6\% $(n=422)$ os instrumentos foram devidamente identificados, em $9,1 \%(n=46)$ das citações, os dados fornecidos pelos sujeitos não permitiram uma identificação adequada do instrumental, e 7,3\% (n= 37) das citações foram referentes às técnicas de Avaliação Psicológica, como por exemplo, observação, entrevistas, desenho livre e jogo de areia.

Os dez instrumentos mais citados pelos sujeitos foram WISC - D. Wechsler (15\%, $n=42)$; Teste de Apercepção Temática Infantil - C.A.T. (formas animal e humana) L. Bellak e S.S. Bellak $(13,9 \%, n=39)$; H. T. P. - J. N Buck $(13,6 \%, n=38)$; Teste Gestaltico Viso - Motor Bender - L. Bender (13,2\%, $n=37)$; Teste de Apercepção Temática - T.A.T. - H. A. Murray $(9,3 \%, n=26)$; Teste de Warteg - E. Wartegg $(9,3 \%, n=26)$; Rorschach $-\mathrm{H}$. Rorschach $(8,6 \%, n=24)$; Teste de Desenhos e Histórias - W. Trinca $(6,8 \%, n=19)$; Teste de Matrizes Progressivas (escalas geral e avançada) - J. C. Raven $(6,4 \%, n=18)$ e Teste de Pirâmides Coloridas - Pfister $(3,9 \%, n=11)$.

Tabela 2. Testes Psicológicos Mais Utilizados Pelos Psicólogos

\begin{tabular}{lcc}
\hline Instrumentos & $N$ & $\%$ \\
\hline WISC & 42 & 15 \\
CAT & 39 & 13,9 \\
HTP & 38 & 13,6 \\
Bender & 37 & 13,2 \\
TAT & 26 & 9,3 \\
Wartegg & 26 & 9,3 \\
Rorschach & 24 & 8,6 \\
Teste de Desenho História & 19 & 6,8 \\
Matrizez Progressivas & 18 & 6,4 \\
Pfister & 11 & 3,9 \\
\hline
\end{tabular}

\section{Discussão}

Segundo a ótica dos psicólogos, observou-se as duas categorias de problemas mais graves foram instrumento e uso. Ou seja, para os participantes, os problemas relativos à construção do instrumento, às normas do instrumento, às características do instrumento e ao uso do instrumento são os problemas mais graves presentes na avaliação psicológica.

Para eles, não haveria problemas se instrumentos novos fossem lançados no mercado, se fossem criados instrumentos que abordassem diferentes tipos de 
construtos, se as amostras utilizadas para a padronização dos instrumentos fossem brasileiras, se fossem realizadas mais pesquisas sobre validade e fidedignidade, se as instruções fossem melhor estruturadas, se os manuai fossem completos, se houvesse instrumentos para avaliar diferentes realidades sócio-culturais, ou ainda, se o custo do material não fosse tão alto.

Tais exigências dos sujeitos não parecem exageradas, já que muitos autores têm falado sobre isso, como por exemplo, Wechsler (1999) que sugeriu um Guia de Procedimentos Éticos para a Avaliação Psicológica, em que consta: "Ao selecionar um teste psicológico, o psicólogo deve:

(...) considerar as características psicométricas do instrumento a ser utilizado, tais como sensibilidade, validade, precisão e existência de normas específicas ou gerais para população brasileira, (...) verificar se o manual do teste possu informações necessárias para aplicação, correção e interpretação dos resultados do mesmo" (p. 136).

Ainda em relação aos problemas graves, os psicólogos justificam também que esses problemas não estariam ocorrendo se os testes não fossem aplicados indevida, indiscriminada e mecanicamente; por pessoal não qualificado, sem critérios; com instruções erradas; se não houvesse erro de avaliação; supervalorização do quantitativo; interpretação generalizada e o uso de um único instrumento como resultado definitivo.

Tendo em vista estas colocações, vale lembrar que “(...) cabe exclusivamente ao psicólogo a responsabilidade pela qualidade da aplicação dos testes psicológicos, sendo esta condição essencial para a obtenção de um resultado fidedigno”, “(...) o psicólogo deverá respeitar rigorosamente as instruções, os exemplos, o tempo e outras orientações que se encontram no manual ou no próprio caderno de teste, evitando quaisquer improvisações que possam comprometer todo o processo de validade do instrumento" (Wechsler, 1999, p. 137).

Em relação aos problemas mais freqüentes, as categorias de respostas que apresentaram maior freqüência foram instrumento e formação. Ou seja, para os sujeitos, os problemas mais freqüentes são os relativos ao instrumento, já discutido anteriormente e os relacionados à formação profissional, às Instituições de Formação, aos órgãos de classe, aos docentes e aos próprios psicólogos.

Os sujeitos consideraram os problemas freqüentes semelhantes aos problemas graves em relação à categoria instrumento. No entanto, no que diz respeito à categoria formação (ver definição de categorias nos Resultados), para os sujeitos não haveria problemas se o psicólogo estivesse melhor preparado para atuar, se o psicólogo não tivesse preconceito em relação aos testes psicológicos, se o psicólogo fosse mais objetivo nas suas avaliações, se o psicólogo fosse melhor formado na área, se os docentes que ensinam nas Instituições de Ensino Superior fossem mais qualificados, se a prática e a carga horária das disciplinas fossem maiores e se houvesse melhor divulgação dos instrumentos para a comunidade de psicólogos.

Mais especificamente em relação ao uso dos instrumentos psicológicos, os resultados deste estudo estão em concordância com o trabalho desenvolvido por Azevedo e colaboradores (1996), no qual objetivou-se conhecer os instrumentos psicológicos mais utilizados pelos sujeitos e identificar a opinião dos psicólogos a respeito dos testes. Constatou-se que metade da amostra utiliza testes como um recurso disponível e possível ao psicólogo; constatouse também que a maioria dos sujeitos acredita no valor dos testes, mas com as seguintes ressalvas: que ele seja usado respeitando os princípios éticos e desde que faça parte de um processo de avaliação, que inclua outros tipos de instrumentos.

Parece estar claro que tais problemas estão sendo atribuídos ao instrumento, ao uso deles e à formação profissional, e por trás disso, encontra-se o psicólogo, ou mais especificamente, o psicólogo que não cria bons instrumentos, o psicólogo que não os utiliza adequadamente e o psicólogo que não está sendo bem formado.

$\mathrm{Na}$ verdade, esta é uma discussão ampla e que envolve muitas instâncias do ser e do fazer psicológicos. Se por um lado existe o psicólogo que está atuando na prática, que não está preparado para as solicitações do mercado profissional e que não está atualizado, por outro lado, tem-se instituições formadoras que não estão formando de acordo com as necessidades profissionais, e por outro lado ainda, tem-se os pesquisadores cujos conhecimentos e descobertas não estão sendo transmitidas de maneira eficientes.

Como pondera Custódio (1996), muitas vezes a "culpa" da avaliação psicológica inadequada e do diagnóstico incorreto é atribuída ao próprio instrumento padronizado. Essa colocação é corroborada por Salvia e Ysseldyke (1991), cujo parecer é de que o instrumento de avaliação é valorizado ou desvalorizado pelo bom ou mau profissional que o utiliza, ou não com adequação, respeitando seus limites e objetivos. Tal afirmação, no entanto, deve ser ponderada, uma vez que não é exeqüível que bons psicólogos transformem maus instrumentos em bons, isentando assim, a má qualidade de alguns deles.

De qualquer maneira, as avaliações ineficientes não podem ser atribuídas unicamente ao instrumento. Não se pode esquecer de que embora o psicólogo disponha de técnicas e recursos para realizar a avaliação, ele tem em sua pessoa o seu instrumento de trabalho, e cabe a ele refletir sobre os dados encontrados. O psicólogo que está 
preparado para isto, certamente estará para compreender os fenômenos psicológicos representados pelos dados numéricos.

A análise crítica dos psicólogos deve ser tão priorizada quanto os dados fornecidos pelos instrumentos, pois eles têm limites e isso não poderia ser diferente, pois em qualquer ciência, os instrumentos têm função auxiliar. A formação e a atualização adequadas do profissional, ao lado de sua experiência, deveriam, evitar tais inconvenientes.

No que diz respeito aos instrumentos psicológicos mais utilizados na prática profissional dos psicólogos, observou-se que grande parte dos sujeitos não os utiliza, enquanto outra parte deixou a questão em branco, o que revela o pequeno compromisso dos sujeitos com o desenvolvimento de sua profissão, considerando que pesquisas científicas promovem o avanço de qualquer área de conhecimento.

A questão solicitava os instrumentos psicológicos mais utilizados na prática profissional e seria aceitável que os sujeitos se manifestassem dizendo que avaliam, mas sem utilizar instrumentos padronizados, mas por outro lado, as justificativas apresentadas caminharam para um discurso antigo, em que o preconceito pelo teste psicológico ainda se faz presente e, em que ainda, o teste é sinônimo de avaliar.

Em relação aos instrumentos mencionados, observouse que a grande maioria tem como objetivo avaliar a personalidade do indivíduo, enquanto uma menor parte objetiva avaliar a inteligência. Estes dados podem ser corroborados pelo estudo promovido por Almeida e colaboradores (1998), já que dos instrumentos mais utilizados pelos psicólogos da Espanha, de Portugal e dos Países Iberoamericanos, oito diziam respeito à avaliação da personalidade. Embora o instrumento da autora deste estudo solicitasse que o sujeito identificasse os testes psicológicos mais usados, alguns psicólogos citaram técnicas de avaliação, o que revela determinada confusão conceitual.

Para Oakland (1996), os testes são fortes em países que valorizam a tecnologia e as diferenças individuais, que tem o sistema educacional bem desenvolvido e que possuem recursos comerciais e tecnológicos para produzilos. Ele argumenta que países sem essas qualidades, geralmente desenvolvem e usam poucos testes e que, ao contrário, a presença das características causa um forte impacto nos testes. Por sua vez, os testes psicológicos de alguma forma representam uma categoria profissional, por serem instrumentos exclusivos dos psicólogos, e é possível que maus instrumentos, aqueles que causam um impacto negativo na comunidade científica e na sociedade, denigram a profissão.
É certo que o estudo não respondeu todas as questões existentes, até porque este não era seu objetivo. Outros estudos na área são necessários, como os relacionados à formação profissional, à comparação da prática da avaliação quando realizada em diferentes contextos, e à concepção de Avaliação Psicológica segundo psicólogos de diferentes formações profissionais, entre outros temas relevantes necessários.

\section{Considerações Finais}

Vale destacar que, embora as respostas dos sujeitos tenham sido divididas em categorias, a fim de se compreender melhor os dados encontrados, na verdade, todos eles têm em comum a ampla questão da formação profissional do psicólogo. Ainda é a formação que dirige a ação do psicólogo e, para que se obtenha uma ação com um mínimo de problemas graves e freqüentes, devese investir na preparação do profissional. É urgente a necessidade de se olhar para isto!

\section{Referências}

Adánez, G. P. (1999). Procedimientos de construccion y analisis de tests psicometricos. Em S. M. Wechsler \& R. S. L. Guzzo (Orgs.), Avaliasãa psicológica: Perspectiva internacional (pp. 57-100). São Paulo: Casa do Psicólogo.

Aftanas, M. S. (1994). On revitalizing the measurement curriculum. American Psychologists, 49(10), 889-890.

Almeida, L. S., Prieto, G., Muñiz, J. \& Bartram, D. (1998). O uso dos testes em Portugal, Espanha e Países Iberoamericanos. Psychologica, 20, 41-55 Anastasi, A. \& Urbina, S. (2000). Fundamentos da testagem psicologica. Porto Alegre: Artes Médicas

Ancona-Lopez, M. (Org.) (1987). Avaliacão da inteligência I. São Paulo: E.P.U Azevedo, M. M., Almeida, L. S., Pasquali, L. \& Veiga, H. M. S. (1996). Utilizaça dos testes psicológios no Basi: Dados de estudo prelimin lzação dos testes psicologicos no Brasil: Dados de estudo preliminar (aches, C. Machado \& M. R. Simões (Orgs.), Avaliacãa psicológica: Formas e contextos, v. IV (213-219). Braga, Portugal.

Bruno, M. L. (1995). Utilização de testes em orientação vocacional. Boletim de Psicologia, 45(102), 81-84.

Custódio, E. M. (1996). Avaliação das dificuldades de aprendizagem: Novas perspectivas para a avaliação psicoeducacional. Em S. M. Wechsler (Org.), Psicologia Escolar: Pesquisa, formação e prática (pp. 157-176). Campinas: Ed. Alínea.

Groth-Marnat, G. (1997). Handbook of psychological assessment. New York, John Wiley and Sons.

Halperin, J. M. \& Mckay, K. E. (1998). Psychological testing for children and adolescent psychiatrists: A review of the past 10 years. Journal of
and the American Academy of Child and Adolescent Psycbiatry, 37(6), 575-584.

Hays, R. \& Wellard, R. (1998). In training assessment in postgraduate training for general pratice. Medical Education, 32(5), 507-513.

Messick, S. (1995). Validity of psychological assessment: Validation of inferences from person's responses and performances as scientific inquiry into score meaning. Conference on Contemporany Psychological Assessment. American Psychologist, 50(9), 741-749. 
Noronha, A. P. P. (1999). Avaliasão psicológica segundo psicólogos: Usos e problemas com enfase nos testes. Tese de Doutorado não-publicada, Instituto de Psicologia, PUC-Campinas, Campinas, SP.

Oakland, T. (1996). Qualities that will influence testin and assessment practices with children and youth toward the beginning of the twenty-first century: International perspectives. Psicologia Escolar Educacional, 1(1), 11-18.

Oakland, T. (1999). Emerging testing and assessment pratices with children and youth. Em S. M. Wechsler \& R. S. L. Guzzo (Orgs.), Avaliação Psicalógica: Perspectiva internacional. (pp. 119-131). São Paulo: Casa do Psicólogo. Psicólogo.

asquali, L. (1991). Relatório do Encontro de pesquisadores em psicometria. Reunião Anual da Sociedade de Psicologia de Ribeirão Preto (texto mimeo). asquali, L. (1992). Avaliação psicológica: Questões e controvérsias [Resumos]. Anais do I ${ }^{\circ}$ Congresso Nacional de Psicologia Escolar (pp. 25-27). ABRAPEE/Campinas - PUCCAMP.

Reynolds, C. R. (1998). Reliability of performance on the test of memory and learning (TOMAL) by an adolescent learning disability sample. Educational and Psychological Measurement, 58(5), 832-835.
Saccuzzo, D. P. \& Johnson, N. E. (1995). Traditional psychometric tests and proportionate representation: An intervention and program evaluation study. Psychological Assessment, 7(2), 183-194.

Salvia, J. \& Ysseldyke, J. (1991). Avaliasãa em educaşão especial e corretiva. São Paulo: Manole.

Wechsler, S. M. (1999). Guia de procedimentos éticos para a avaliação psicológica. Em S. M. Wechsler \& R. S. L. Guzzo (Org.), Avaliação psicologica: Perspectiva internacional (pp. 133-141). São Paulo: Casa do Psicólogo.

Witter, G. P. \& David, J. B. (1996). Avaliação da Pós-Graduação na PUCCAMP: Auto e hetero-avaliações. Estudos de Psicologia, 13(2), 3-10

Zimiles, H. (1996). Rethinking the validity of psychological assessment. American Psychologist, 51(9), 980-981.
(19)

Sobre a autora

Ana Paula Porto Noronha é Docente da Graduação em Psicologia e do Programa de Estudos Pósgraduados em Psicologia da Universidade São Francisco (USF). Doutora em Psicologia: ciência e Profissão pela Pontifícia Universidade Católica de Campinas (PUC-Campinas), Campinas, SP. 